\title{
Erratum to: An Orchestrated Security Platform for Internet of Robots
}

\author{
Mehrnoosh Monshizadeh, Vikramajeet Khatri (D), \\ Raimo Kantola, and Zheng Yan
}

\section{Erratum to: \\ Chapter "An Orchestrated Security Platform for Internet of Robots" in: M.H.A. Au et al. (Eds.): \\ Green, Pervasive, and Cloud Computing, LNCS 10232, https://doi.org/10.1007/978-3-319-57186-7_23}

The original version of this chapter unfortunately contained a mistake.

The ORCID identifier for the second author, Vikramajeet Khatri, was missing.

His ORCID is: 0000-0002-3386-8952. 